\title{
L'épée inscrite de Boston (K. 1048, 1040-41 de notre ère)
}

\section{Gerdi Gerschheimer, Brice Vincent}

\section{Abstract}

A gilded bronze sword, kept in the Museum of Fine Arts of Boston (Massachusetts) since the beginning of the 1970s, bears a short inscription of two lines in Old Khmer. A new reading of the inscription allows one to correct previous publications. A wrong reading, among other factors, has led to date the sword 862 saka/ 940 AD (under Jayavarman IV), and thus to provide "hard evidence for [the] use [of mercury-gilding for Khmer artifacts] during the tenth century". In fact, the inscription clearly bears the date 962 saka/1040-41 AD (under Suryavarman I).

\section{Citer ce document / Cite this document :}

Gerschheimer Gerdi, Vincent Brice. L'épée inscrite de Boston (K. 1048, 1040-41 de notre ère). In: Arts asiatiques, tome 65, 2010. pp. 109-120;

doi : https://doi.org/10.3406/arasi.2010.1708

https://www.persee.fr/doc/arasi_0004-3958_2010_num_65_1_1708

Fichier pdf généré le 08/11/2019 


\section{Gerdi Girschheimfr et Brice Vincent}

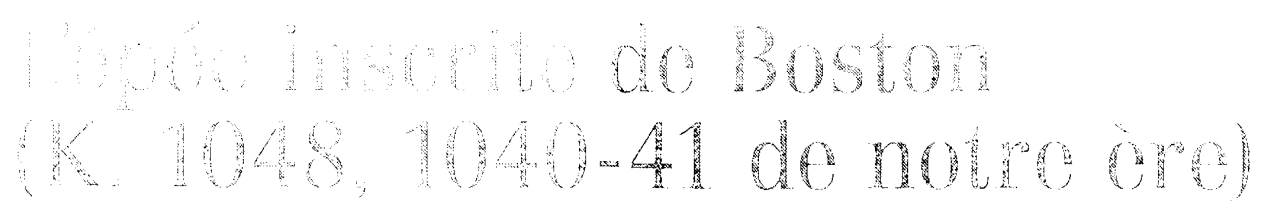

Parmi les pieces présentées en 1982 dans le catalogue Asiatic Art in the Museum of Fine Arts, Boston figure une épée courte en bronze inscrite de deux lignes en vieux khmer' (fig. 1). La notice propose une traduction de l'inscription (voir infra note 10) solon laquelle l'épée a óté offerte en 964 śaka, c'està-dire en 1042-43 de notre ère, quand Süryavarman I"r (r. 1002-1050) régnait sur le Cambodge?2. I.intérêt pour cet artéfact et son inscription a été renouvelé en 2004 par leur publication dans louvrage consacré par limma C. Bunker et Douglas latch ford à « lîge d'or de l'art khmer $»^{3}$. Ils y donnent une lecture de linscription (sans traduction, mais avec: une paraphrase partiellel proposée par le regretté Professeur Long Seam, sur la base dune copie manuscritet. Mais la

* Vinus remercions en particulier Clande dacques, toujours prompt à partager ses domnées ort ses connaissancess, le Museum of line Arts de Boston tri-apres MEVI. et Laura Woinstein. Ananda Commaraswamy Curalor of South Asian and Islamic Ar: du MLA, qui nous a gincereusement ouvert ses archives.

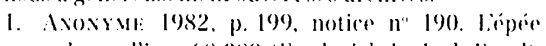
porte Ir " I" d'ins. 68.289 (lirederick I.. Jack liund).

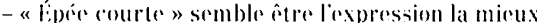
adaptio pour qualifier cet objet. qui a jusqu à prósent recu plusieurs denominations difterentes dans les diverses publications le concernant. Vuir infra p. 114.

2. Cinst aussi la datation qui figure sur létiquette prisentant arctuellement (nowembre 2010 lópere au IIF : voir infra note 10 .

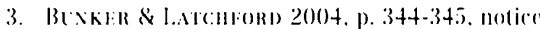
n" 122. Sur cel ouvage, wore le compte rendu de

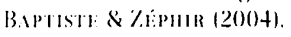

1. Pour la copie de l'inticription. voir alussi Bexkek

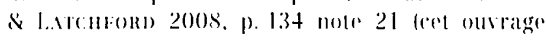

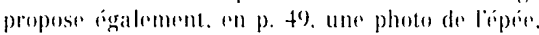

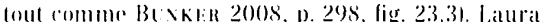
Wreinstein a bien voulu nous rommuniquer une copie d'un document manuserit qui est assurement relui qui a folc utilisei par long som

Illustration non autorisée à la diffusion

Pig. 1 : « Fée de Boston ». Provenance incomnue. 1040-41 dé n. ì. Bromze doré au mereure. l. $23 \mathrm{~cm} \mathrm{et} 1.8 \mathrm{~cm}$.

VI:A (68.289. Froderick L.. Jack liund). (photographie MIA).

date y est lue 862 saka (940-941), sous le ròne de Jayavarman IV. Cette épée dorée au mercure fournirait ainsi « the earliest dated evidence for the use of mercury-gilding in Cambodia. Mercurygilding is present on numerous later Khmer artifacts produced during the eleventh century, but hard evidence for its use during the tenth century has been lacking $»^{\bar{x}}$.

l'inscription de l'épée de Boston avait été intégrée dès 1971 à l'inventaire des inscriptions du Cambodge sous le numéro K. 1048". La date on avait été lue « 964 saka », soit 1042-43 de n. è. Cette donnée s'appuyait sur une photographie asse\% nette de l'inscription que Jan Fontein, alors conservateur du MFA, avait envoyée en 1971 à (laude Jacques (fig. 2). Ce dernier lui avait proposé, dans un courrier en date du 23 juin 1971, une lecture et une

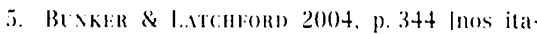

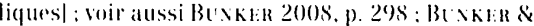
J.ATCH:ORD 2008 . p. 45.
}

6. Jovers 1971.p. 18.3. raduction de l'inscription, qui concordent tans les grandes lignes avec celles que hous proposons ici. C"est très probablement sur la base de cette lecture, ou sur elle que Son Soubert avait communiquée Jan liontein dans un courrier en date du 24 mai 1971, que le catalogue de 1982 a adopté la date de 964 śaka

D'une longueur de $23 \mathrm{~cm}$ et large de $8 \mathrm{~cm}$ au niveau do la gardes, l'épée a été réalisée par le procédé de fonte à la cire perdue, très vraisemblablement d'une seule pièce, puis entièrement dorée au mercure (BUNKI:R \& IATCIIVORI) 2004, où l'objet est décrit avec précisionı. Il faut sans doute renoncer à l'espoir de jamais connaître son lieu d'origine : «Quoique la provenance exacte de notre poignard ne soit pas connue, j’ai obtenu, par hasard, quelques informations sur son origine. Il paraît que le poignard faisait partie d'une collection d'objets d'art d'un intérêt artistique assez modeste. Un marchand d'art à New York avait acheté la collection complète, mais quand les pièces arrivèrent à New York le poignard avait disparu. Quelques mois plus tôt nous l'avions acheté à Londres. (I a possibilité de l'existence de deux poignards ne peut pas être rejetée sans question.) » (Jan Fontein, lettre datée de Boston, le 2 juin 1971, adressée à (Claude Jacques). Aussi bien le catalogue de

7. Le courrier de Son Soubert, que nous a communique Latura lleinstein. est alecompagné doune Ereture et d'une traduetion francaise apparemment dues a claude Jacques. Ia traduction differere de celle proposép le 23 juin.

8. Cost sans doute a la suite d’une coquille que le ratalogue de 1982 donne une longueur de $28 \mathrm{~cm}$ 1.Nowswe 1982. p. 1991. 
Fig. 2 : I.inseription K. 1048. (photographie ML:1).

1982 donne-t-il prudemment la provenance comme étant " Cambodia or Thailand" ( Nonyme 1982, p. 199). ()n ne voit pas sur quoi se fondent JAcQuis (1971, p. 183) d'une part, BunkFr \& IATCHFORI) (2004, p. 344) de l'autre, pour affiner cette donnée et proposer une origine, respectivement, en Thaillande ou au Cambodge.

\section{Texte}

linscription de deux lignes (en fait une ligne et demie) qui figure sur l'un des plats de l'épée en explicite la destination. Tant la souplesse que la largeur et la profondeur des caracteres qui la composent invitent à penser qu'ils ont été directement gravés sur le modèle en cire, avant la fonte de l'objet. Notre édition est fondée. sur l'examen du cliché communiqué en 1971 à C. Jacques par le $\mathrm{MF} \Lambda$ (voir fig. 2)" :

\section{(1) 962 śaka jalm/nvan· loña 'yak can hvara ta müla}

$962: 964$ haceues 1971. Anonyme 1982 : 862 BUNKER \& I ATCHFORD 2004. ja/mlnvan : jamnean BUNK I:R \& LATCHFORD 2004 looquille: jamnean dans leéd. en car. khmers).

\section{(2) ta kamraten - jagat(a) treh} jagat(a) : jagal (a BUNKr:R \& I.ATCHFORD) 2004. - Il est possible quïl y ait un virāma sur le I de jagat. Il est par contre certain. qu'il n'y a pas de ta après jagal(a).

treh : tre... BunKe:R \& I.ATCHFORD 2004. où il est de surcroît proposé de restituer cet élément en traibhüraneśsara.

Soit, dans la traduction que nous en proposons :

« 962 śaka, offrande de Loñ 'Yak (de) Canhvar, müla, au Kamraten Jagat (de) Treḥ. "10

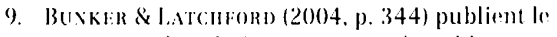
texte (en caracteres latins at en caracteres khmers). Vous indiguons les différences de lecture : olles sexpliquent en partie par le caractiore défectueux de la copie manuscrite (ef. supre note t) dont a dispose long seam.

10. Voicei les traductions anglaise's dont nous avons comnaissance : "In the year 964 Śaka (A.D). 1042) lon lyak Canhvar from the monastery of Kamratin Jagat Treh made this offering " Axowsme 1982.

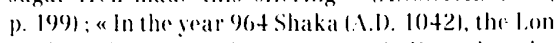
Irak Canhar from the sanetuary dedicated to the Sorereign of the Iniverse of Treh made this offo-

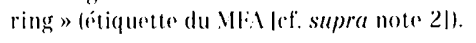




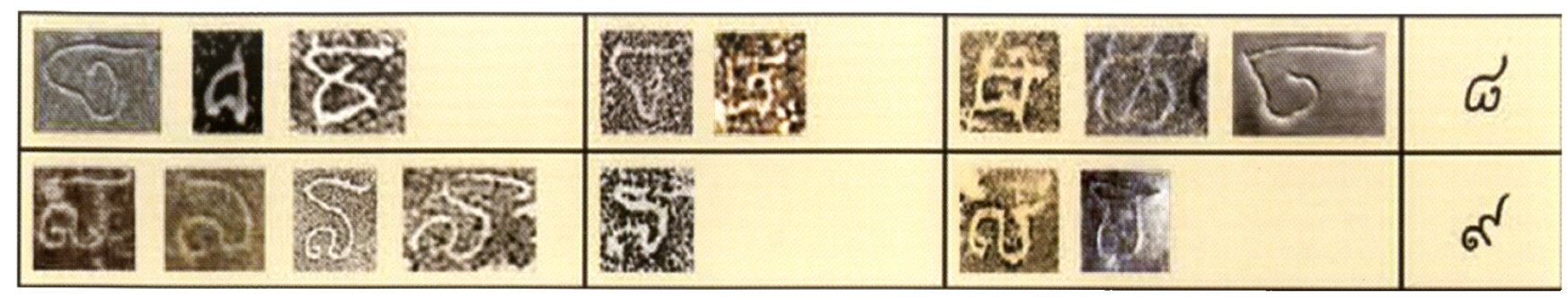

Jig. 3 : Tableau de numeration khmere pour les chiffres 8 et $9\left(x^{*} \times x^{\prime}\right.$ siectes). Id apries Soutri: 2008 , ill. 13 p. 76 ).

\section{Date}

Éliminons tout de suite la lecture du chiffre des unités comme un « 4 », manifestement erronée". Le chiffre des dizaines est assuré, et le débat porto sur celui des centaines. $A u$ vu du cliché publié ici (fig. 2), il s'avère que la copie manuscrite dont disposait long Seam était inexacte : le chiffre des centaines de K. 1048 ne peut être 8 (fig. 3), et il est du même type que le chiffre des centaines dans des inscriptions telles que le fameux «serment des fonctionnaires » de Sūryavarman $\mathrm{I}^{\mathrm{rr}}$ ou K. 1198 , du règne de

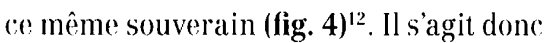
bien d'un 9, et nous sommes alors en 962 saka (1040-41 de notre ère), quand règne au Cambodge Sūryavarman I"r.

La suite de l'inscription pose un certain nombre de problèmes d'interprétation : syntaxique avec les deux occurrences de la difficile particule ta: sémantique avec le terme müla, dont le

11. Biren que la distinction entre les chiffres 2 at 3 soit parfois délicate dans l'ériture khmore. il ne semble pas qüil laille retenir ici la possibilite de lire un « 3 " plutôl quiun « 2 ».

12. C"est loceasion. incidemment. d'insister sur limportance de reproductions do qualiti lestampages on photographies) des inseriptions, en parliculier quand un examen direel de lorighinal nest pas possible: le meilleur philologue ne peut guirere aller bien all-dela des documents dont il dispose. sens est mal circonscrit ; sans compter les habituelles difficultés que présentent les anthroponymes ou les toponymes.

\section{Donateur}

Selon Bunker \& Iatehford, l'inseription nous apprendrait que " the king gave this dagger to Lon Ayak $»(2004$, p. 344). (On ne voit cependant pas qu'aucun roi soit mentionné, et la séquence jamnoan $X$ la $Y$, jusqu'à plus ample informé, signifie " offrande / don de X à $Y{ }^{13}$. Le donateur est donc: un Loñ.

13. In voici quelques exemples, dans des épigraphes inscrites sur des objets en métal «mobiless": - 929 saka jamnvan ka/m/mratena 'aña trah cau la rrah kammraleria 'aña śiralinga thmo reaka (...) : "929 saka : don du Kammratein Dñ Vrah Cau alu Vrah Kamraten Xn Sivalinga (de) Thmo Vrak (...)" (K. 1218 , inscription sur un vase en bronze, che\% un antiquaire de Bangkok en 2006 : texter trad. dans So(tTt: 2009 , p. 598$)$

- los9 saka rrah jammvan trah päda kamrateria añ śri tribhuranādityararmma/dlever la $/ \mathrm{kat}$ mrateria jalglata chpara ransi : " loke sake :

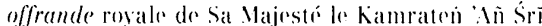
Tribhuranădityasarmadeva call Kamraten Jagal (de) Chpar Ransi $" 1 k$. 1219. inscription sur un vase en bronze deune collertion privere a Bangkok: texte et traduelion dans So(tal 2009. p. 606).

- ja!nunan tral kamratén añ śri rivendrädhipatirarmma la kamrateri jagal sriciressara na samrok ta llog saka : "Offrande de V. K. . L. Sī Virendrädhipativarman all K. J. Śri Vireśsara dans Sambok on 1109 s. " (K. [055.1. inseription sur un bol en bronze. trouve sur to site n" I1 do

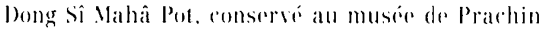
Buri|214/252+1 : texte el trad dapres condes dans Bolssit.IFR 1972, p. 471.
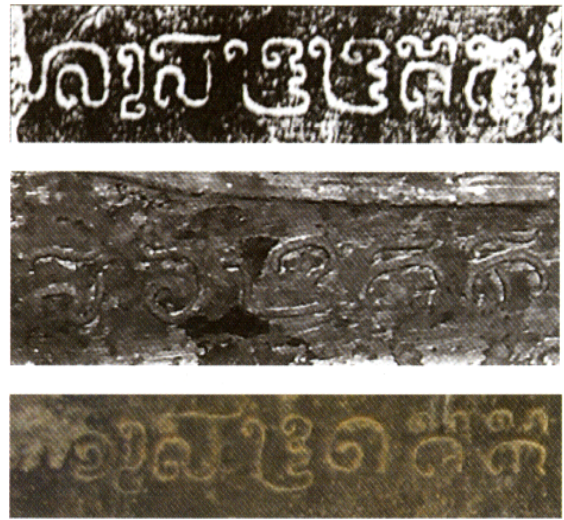

Fig. 4 : 1)étails de dates dinscriptions contemporaines de K. 1048 . a. K. 292 (« Serment desi fonctionnaires") 933 śaka.

b. K. 1048 : 962 saka. (détail de la fig. 2) c. K. $1198: 931$ saka.

« monsieur, le sieur» (Pou 2004, p. 422b, s. v. loñ), portant ce " title of an unidentified rank or function » (Ji:NNer 2009 , p. 513, s. v. loñ) extrêmement fréquent en khmer angkorien ${ }^{14}$. Il ne s'agit certainement pas d'un rang ou d'une fonction très élevé, et c'est peut-être là un des traits les plus intéressants de cette inscription de donation d'un objet, comparéc aux autres épigraphes du même type, où les donateurs sont le plus souvent de haut rang (cf. supra note 13 ).

- II.3s saka rrah jammana rah pada kamralena aña sri jayararmmadera ta rrah lokeśserare : «1138 saka offrande royale de Sa Majeste le Kamraten An Srī Jayavarmadeva I= Jayalarman VIII all vénerable I.okeśrara " (K. 1251. inscription sur un bol en argent conserví all musér national du Cambodgre. Phnom Penh lga 6906) : texte el trad. dans Sol.1. 2009, p. 61:3).

- Voir aussi. dans a mêmo volume. l'inseription k. 1277 trattée par ). Soutil'(p). 1221.

14. Javer 2009. trés laconique sur loñ. prérise

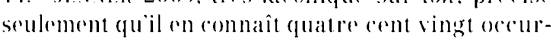
rencess en khmer angkerien. 
'Yak est un anthroponyme très commun en khmer angkorien (JinNNER 2009 en connaît 32 occurrences). Commex pour nombre d'anthropenymes, une recherche exhaustive vaudrait d'être entreprise pour déterminer sa date d'apparition dans le corpus ${ }^{15}$. La fréquence même du nom propre est peut-être cause quon le spécifie parfois par un nom de lieu ${ }^{16}$, comme c'est très probablement le cas ici : « I.oñ 'Y. du Canal / de la Rigole / du Ru $»^{17}$.

La présence, après le " nom » du donateur, du relateur $t a$ pourrait induire à croire que ce dernier introduit le donataire, le second ta servant alors à déterminer le terme qui précede : " don du I.oñ Yak Canhvar au müla de K. J. Treh ». Mais qu'est-ce qu'un müla? Le terme, importé du sanskrit müla, est difficile ${ }^{1 *}$. Nous ne pensons pas, cependant, quiil puisse signifier « monastery » ou « sanctuary », comme l'entendent le catalogue de 1982 ou l'étiquette du musée (cf'. supra note 10), qui comprennent du reste d'une. façon encore différente les deux ta de l’inscription ( « don du Loñ Canhvar du müla de K. J. Treh »). Restant dans l'hypothèse où le premier ta introduirait le donataire, on pourrait imaginer que müla

15. Il ne semble pas que le mot soit atteste dans des inseriptions antérieures au règne de Javavarman $V(r$. $968-100(0)$ - mais les portions khmeres do bon nombre d'inseriptions de koh ker sont encore innidites... Toutefois, selon la partie angkorienne de $\mathrm{k} .956, \mathrm{x}^{*}$ siecte ?I, une Ten 'Yak (I. 9. /.' VII. p. 130) est suppo-

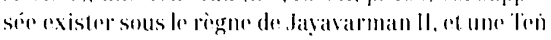

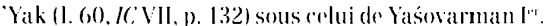

16. (On a ainsi, dans les inseriptions de Banteay

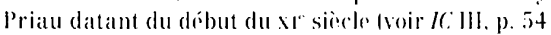

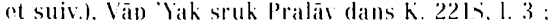
Vāp Yak Samgramapúra dans K. 221 V. 1. 9) : I.oñ Yak Nāgapura ot Vāp Yak Chdio Jrau lu V. 'Yak dr Chdio Jrau (" riviere profonde ") m, KC' III, p. 6.3) dans k. $222,1.2$ a 1. 13.

17. Sur cenherer, voir Pou 2004, p. 157b et 605a : Jaxver 2009. p. 112-113 : voir aussi infret.

18. Voici les cquivalents quin propesent, respereliwe ment, Saveros Pou (2004, p.377al) of Pluilip X Jemere

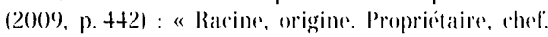

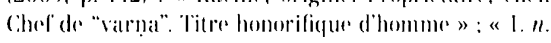
Root: stock. butt. 2. n. Base. bontomn. foot, sole: basis. ground, foundation. 3. "n. Beginning, sourere. origin. gemesis. 4. n. Stork lof chattelst, force, store, fund, rapital: substance. wealth, estate, property: worth, value. 5 . n. Principal, ehief, leatder, loading member: landowner. landed proprietor ». (Nous ne tenons pats compte ici d'un second mül müla enregistrí patr denner.)

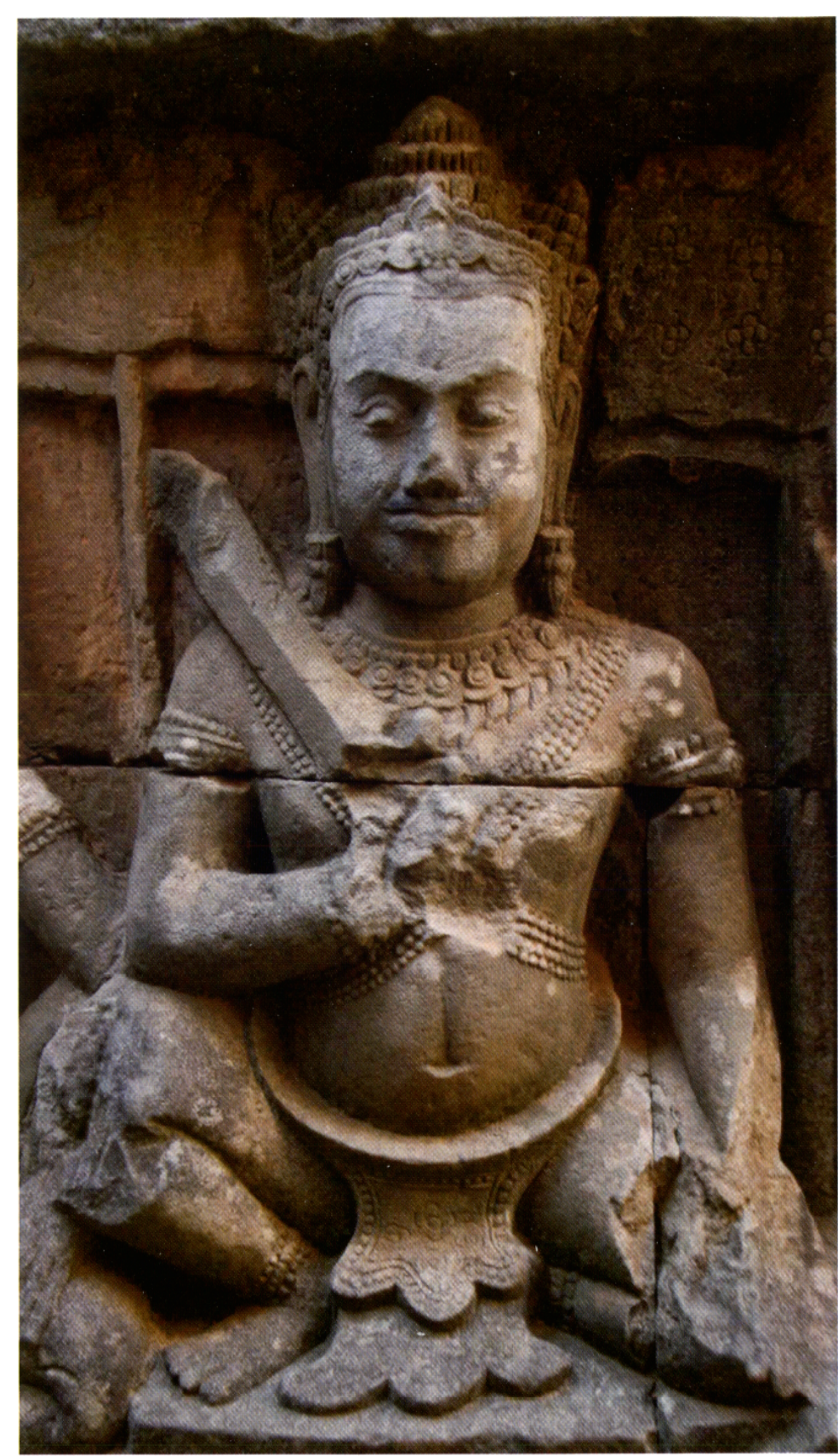

Pijg. 5 : Yakșa (?) tenant une épée courte.

Reliefs interieurs de lat Terrasse du Roi leppreus. Angkor Thom (provinee de Siem Reap) län du xu" - début du xul" siecle (?) Cirès.

Ipholographir B. Vincent 2010). 


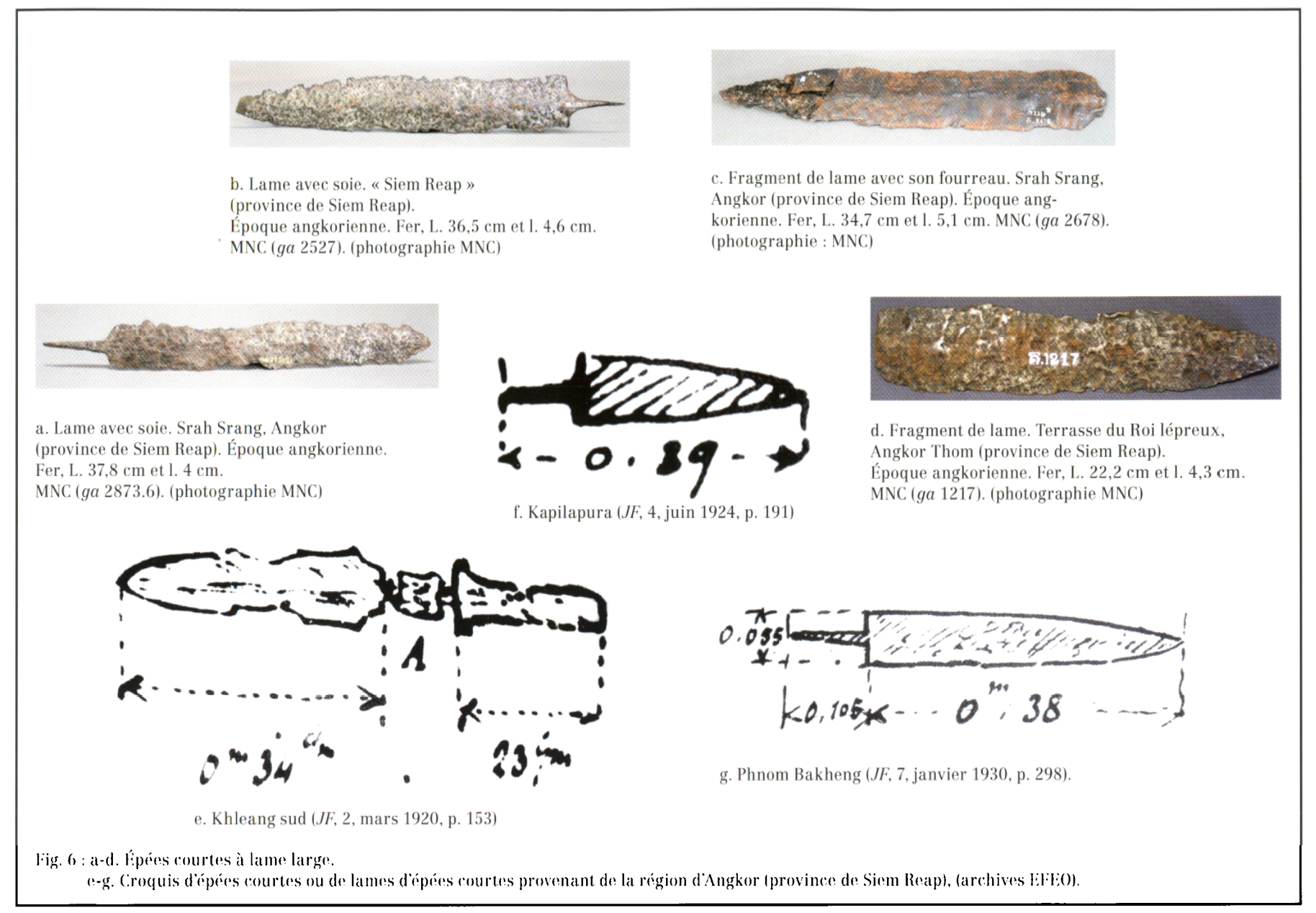

soit à prendre en son sens de « estate, property » : « don du Loñ 'Yak Canhvar au (ta) capital (müla) du (ta) K. J. Treh ».

Mais on ne donne pas à un "capital» : on l'accroît. On doit donc considérer que c'est le second $t a$ qui introduit le donataire, et que le premier ta introduit une détermination du donateur : "Loñ 'Y. C.., qui est müla», «principal » en un sens très général du mot. Dans l'attente de nouvelles enquêtes sur ce terme, rappelons celle que lui a consacrée Claude
Jacques (1977-78, p. 1073-1075), d'où il ressort que müla pourrait désigner ici " une sorte de fondé de pouvoir, gérant une propriété - un capital foncier appartenant à sa propre lignée " (ibid., p. 1074). C'est alors du contexte qu’il faut extraire le corrélatif implicite de ce terme : il s'agit probablement du capital foncier du sanctuaire de K. J. Treh, peutêtre un temple de lignée.

linfin, on ne saurait exclure la possibilité que nous signale Michel Antelme.
Il est en effet étrange de spécifier l'anthroponyme 'Yak par un terme générique tel que canhvar (voir supra) : de quel ru s'agit-il ? Il se pourrait bien que ta müla soit ici à lire en un seul mot, tamüla, et soit le nom du canhvar en question! Notre donateur serait alors le I.oñ 'Yak du Canhvar Tamūla. Nous laissons aux linguistes le soin de déterminer quels pourraient être l'étymologie, le sens et les dérivés, en khmer moderne, de cet hapax $\left({ }^{*}(m \bar{u} l)\right.$. 


\section{Donataire}

Le donataire est donc: le « K. J. Treh », une divinité appartenant à la catégorie maintes fois étudiée des Kamraten Jagat (" High lord of the World»)". La restitution - ou émendation- raibhüeaneśseare proposée, via Long Seam, par Bunker \& I.atchford (2004, p. 344) permettrait d'échapper au difficile mot treh. lille est cependant improbable au vu du visarga (h) clairement grave apres la syllabe tre (qui du reste ne peut être lue trai). II semble par ailleurs tres douteux, au vu des clichés dont nous disposons, que la seconde partie de la deuxieme ligne ait jamais été inscrite.

le terme treh. avec ses tries probables variantes (tareh. treh), est extrêmement rare dans l'épigraphie, et son sens est mal établi²0. En réalité, le "sens" de ce terme quand il nest pas un nom propre nous importe peu dans le présent contexte"21 : il nous faut savoir šil sagit. icei d'un anthroponyme (« le K. J. lappelél Treh ") ou d'un toponyme (« le K. J. |del Treḥ ») - et même cette différence est peut-être de peu d'importance. Un survol empirique de paralleles nous incite à privilégier la traduction « (de) Treh » : une étude détaillée de la nomination des K. J. reste à faire.

19. Joxian 2009. p. 2s. Sur les hammiled Jagat.

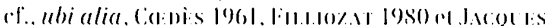
1994

20. Ventionnons seulement les occurrences connues en virus khmer, ell altendant qun des linguistes approfondissent la question : lreh est lo nom d'un resclawe massulin leci) dans $K$. 14\%, I. 7

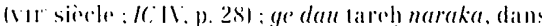

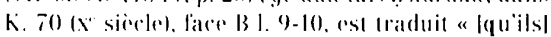

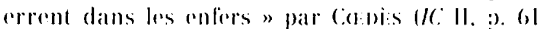

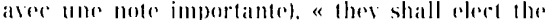
hells " par Jommer (20)9. p. 217 : anlin, lo khlon

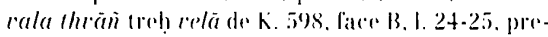
mier tómoin den bornage efferelue le 25 mai 1006.

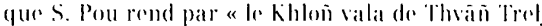

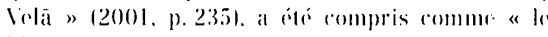

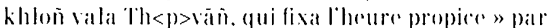
fivoT 11928. p. 77) ou " the commandant of thein. who determined the Ipropitious| hour " par It: vive 1200\%. p. 217 .

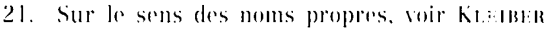
1981.

\section{Une reproduction d'épée courte}

la forme générale et surtout les dimensions de l'objet éludié expliquent sans doute qu'il ait été identifié comme un " poignard " (dagger) dès son entrée dans les collections du muscée de Boston, un qualificatif qu’il conservera par la suite. Cependant, en le comparant aux armes figurees sur les bas-reliefs do l'époque angkorienne, en particulier sur les grands ensembles des xil"-xin" siècles (bas-reliefs d'Angkor Vat, du Bayon et de Banteay (hmar), certes plus tardif's mais d'une iconographie plus riche, il semble? davantage à rapprocher de la catégorie des épées. George Groslier (1921, p. 91-92. fig. 55-A et E) et Michel Jacq-Hergoualc ${ }^{h}$ (1979, p. 41-42, 45, doc. 13.5-6) s'accordent pour reconnaître en elles des armes à double tranchant, généralement courtes et pointues, avec: une lame plus ou moins large, représentée nue ou dans son fourreau, et une poignée diversement ornée. Ces ćpées auraient moins servi darmes oflensives que de marques d'honneur, au même titre que les parasols, étendards. ćventails ou encore enseignes, aussi figurés sur les bas-reliefs. lilles se retrouvent ainsi entre les mains de chefs militaires. tout en étant des insignes de la dignité royale.

D'autres exemples encore d'épées. toujours courtes mais de diflérentes formes, sont sculptés en plus grand format sur les haut-relief's décorant les parements de la Terrasse du Roi lépreux (extérieurs et intérieurs) et du cirand bassin du Palais Royal, à Angkor Thom. l'une dentre elles, tenue par un yaksa. doit particulierement retenir notre attention : même si elle na pas été conservón dans sa totalité - sa poignée est brisée-. elle se distingue par une lame quasi identique à celle de lépée de Boston. Celle-ci est large et à deux tranchants, séparés par une crête médiane, et se rétrérit légerement dans sa partie centrale avant de se terminer en pointe fig. 5 : voir aussi Rovi:DA 2005: fig. 5.17). Une similitude de forme existe done, mais une estimation approximative de la taille de cette ćpée sculptéc lui donnerait toutefois une longueur comprise entre 35 et 40 ('m, soil largement supérieure à cells? de l'objet cétudić. cette simple observation laisserait penser que l'épée de Boston ne serait en réalité qu'une reproduction en taille réduite d'une de ces épées courtes. essentiellement documentées par des sources narratives.

Pour la plupart inédits, quelques exemples d'épées courtes angkoriennes ont néanmoins été mis au jour au Cambodge"2. Plusieurs dentre elles proviennent, en particulier, de la région d'Angkor : certaines sont conservées au musée national du Cambodge à Phnom Penh, alors que dautres ne sont connues que par la documentation archéologique, où elles apparaissent sous lo nom de "glaives". Seule leur lame en ler a eté conservée, entiere - avec leur soicet éventuellement leur poignéc - ou fragmentaire. Celle-ci est toujours à double tranchant symétrique, aver une longueur généralement comprise entre 35 et $40 \mathrm{~cm}$ pour une largeur d'environ 4 à $5 \mathrm{~cm}$ (fig. 6a-g) ${ }^{23}$. Un second type d'épées courtes serait également attesté, même si là encore il n'est

22. Serule uno retude lypologigue sommatire est in proposere sans quane recherehe systematique n'ait ite faite dans le vorabulatire propre alu vieux k hmer pour retrouver des tremes correspondant atux dillírents lypes identifiés.

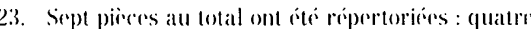

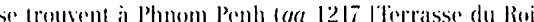

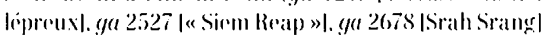
- la serule publicer. mais sans illustration. dans cot kBss

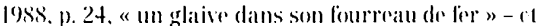

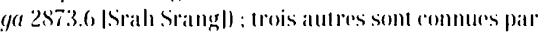

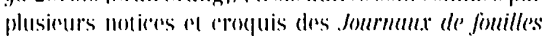
1.11: 2. mars 1920, p. 153 /khleang sudl. " un ghaive tris abime par la rouille. L a lame a 2 tranchants aves nersure mídiane a dauns sil partie lia plus large (0)"

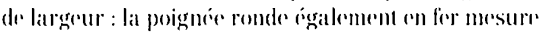

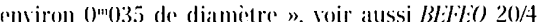
$|1920|$. p. 220 : . $/ 2: 4$. juin 1924, p. 191 (Kapilapural. "une lame rongés par la rouille en lorme de glaive" vir aussi $R(C 4$, juin 1924 : .J: . 7. janvier 1930. p. 298

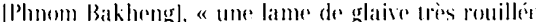
(1)"3s de longuever sur 5.5 millimides de plus grande largene conserves a phuom Penhe mais malheureusement

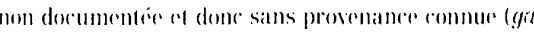
1226). Janfin. lant par sa forme que par ses dimensions.

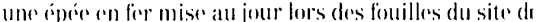
Proi Nonti. toujours dans la région dingkor. sinstoril.

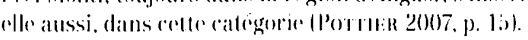




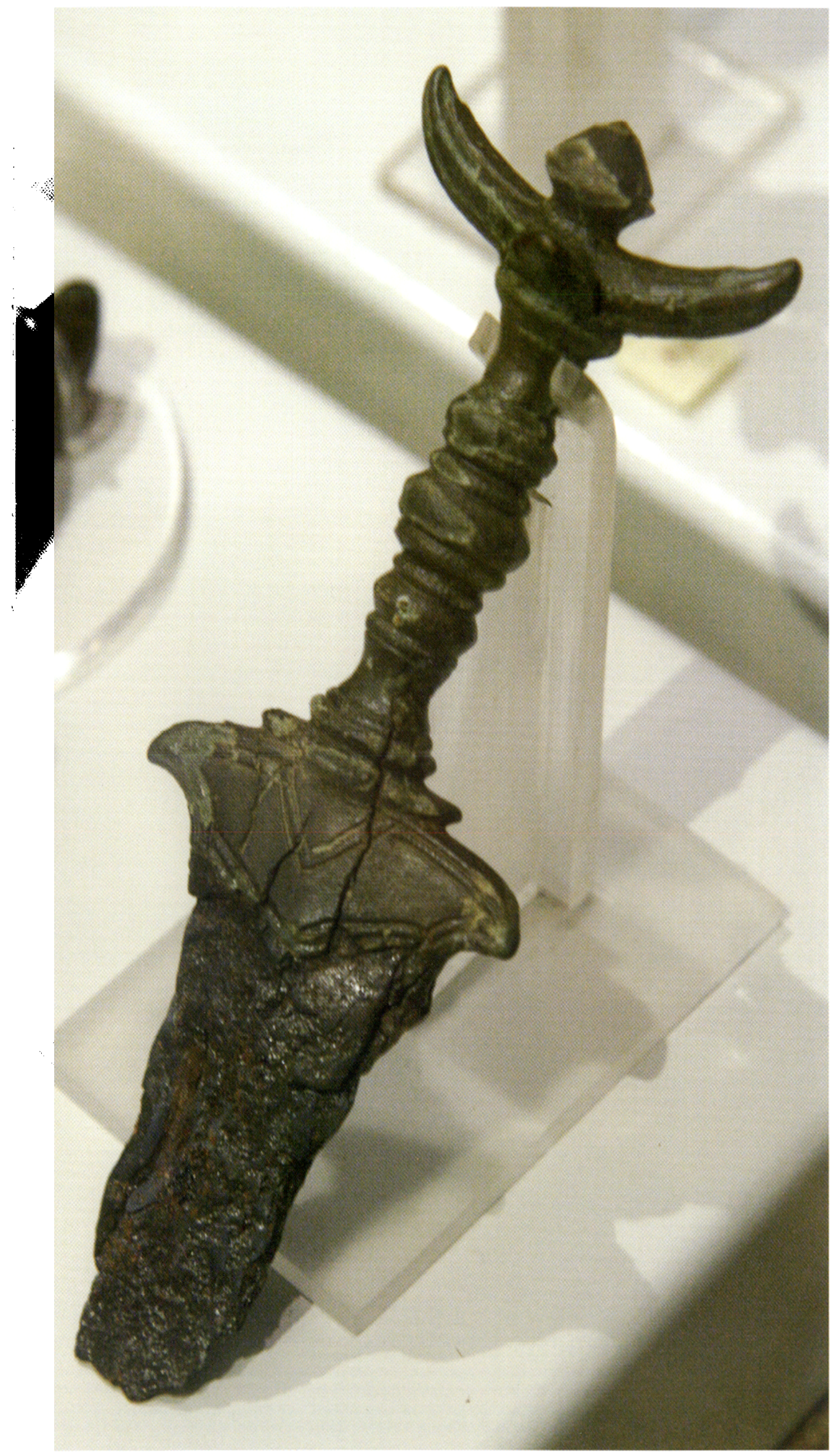

Figs. 7 : Reproductions déperes courtes : attributs de statue ou armes votives (?)

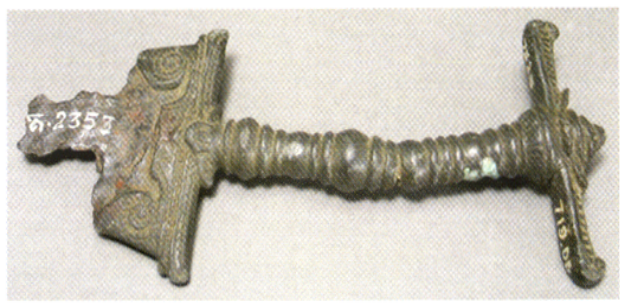

a. Phnom Bayang (prowinee de Takeol. xul"-xur" siecle. Bronze, I. $2.3 \mathrm{~cm}$. MNC (ga 560.5). (photographie MNC.)

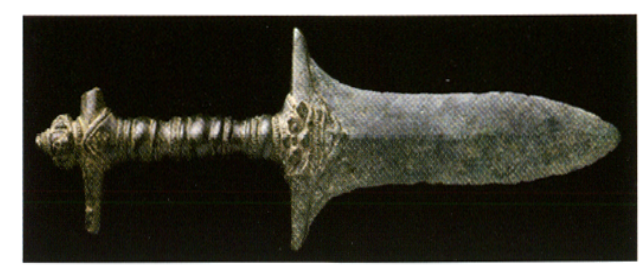

b. Provenance exacte inconnue (rógion d'Angkor ?). Ḱpocque angkoricmne 1?). Bronze et fer. I. 14 c'm. MAC (qe 23533). (photographic MNC)
1. Provenance exacte ineonnue

(provines de surin ?.) lpogue anghorienne (?) Bronze of for. I. 14.5) (mo

Musér national de Surin $(45 / 25+41$.

(photographie B. Vincent 2010$)$ 
représenté que par quelques exemples, certes mieux conservés, issus de collections muséales et privées. Par rapport aux pièces précédemment décrites, celles-ci sont plus courtes, entre 30 et $35 \mathrm{~cm}$ de long au total, avec une lame en fer également à double tranchant mais plus effilée ainsi quiune poignée en bronze cylindrique que complete un pommeau ${ }^{24}$

l'épée de Boston correspondrait par sa forme au premier type d'épées courtes à lame large. Cependant, tant la taille de cet objet que le matériau précieux dont il est constitué - le bronze, lui-même doré - confirment clairement qu’il n’a jamais (ité lonctionnele ${ }^{25}$. Il en serait de même pour une autre épée courte en bronze, elle aussi de taille réduite mais plus tardive (XII"-XIII" siècle), découverte dans le sud du Cambodge, au Phnom Bayang, et aujourd'hui conservée au musée de Phnom Penh (ga 5605 : fig. 7a $)^{26}$. I:nfin, il laudrait rapprocher de ces dernieres deux autres pièces du même typo - leur lame. partiellement conservée, est toutefois en fer-: l'une so trouve aussi à Phnom Penh (ga 2353 ; fig. 7b), l'autre au musée national de Surin (45/2544; fig. 7e). Ces quatre épées répondaient sans doute à une même fonction quill reste encore à déterminer.

24. Ces raractóristiques morphologicues axpliquent sans doute les différentes dénominations quont recues ces pieces quil semble pourtant possible de classer dans cette même caltégorio de's eperes courtes. lime dienter olles est aujourd"hu

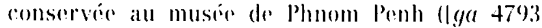
Marcial. 1939. p. 144. " sabre" : Bolssitlon 1966, p. 343. "ripee 》 : deux autres sont au muse

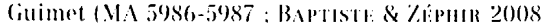
p. $366-367$, notice n" 113 , "poignards "1 : enfin une derniere se trouve dans une collection privé

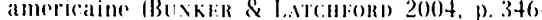
347. notice 12" 123, " short sword $\#$. Ce second type

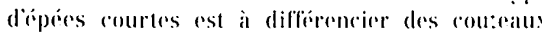
angkeriens, dont la lame est. colle alussi, en for mais plus courte. line premiere rutude do ces couscatux a sté récemment mence par Pierere Bâty $(2007)$ à partir de plusieurs sperimens dicouverts lors des fouilles do lariroport de Siem Reap. a Angker. et dattés des xi"-xil" sièrles.

25. Lidentifieation de la piesere romme un \& poighard crímoniel " ou "rituel " (ceremonial dag-

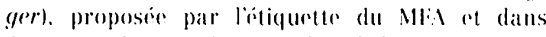
Avoxyme 1982 (p) 199), est done a coarter

26. 181:1\%O) 36 (1936), p. 626, pl. (:-13 : Bolssil.11:R

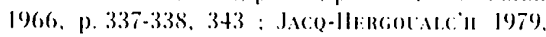

p. 45 : DA.SHEIMIE 2001. p. 280 . notice n" 162

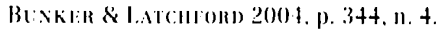

\section{Attribut de statue ou arme votive?}

Jean Boisselier avait déjà proposé d'identifier l'épéc du Phnom Bayang non pas comme une arme, mais comme un attribut mobile de statue, une hypothese qui pourrait également être envisagée pour l'épée de Boston. Encore reste-il à déterminer de quelle divinité elle était l'attribut. lin se fondant sur des témoignages plus ou moins contemporains, tant épigraphiques qüiconographiques, le choix porte sur plusieurs d'entre elles, même si le dieu Viṣnu reste sans doute à privilégier. lin effet, si le disque, la conque, la massue et la Terre comptent parmi ses attributs traditionnels, l'épée (sanskrit khadg $g a^{27}$ ) est, elle aussi, parfois portée par la divinité (BHattacharya 1961, p. 103-104. 107). Viṣnu apparaît par exemple avec: une épée dans sa main supérieure droite. alors que sa main supérieure gauche tient lo disque et ses mains inférieures le serpent Vāsuki, sur le fameux bas-relief représentant le Barattage de l'Oćan à Angkor Vat (galerie est, aile sud ; voir Roveidn 2005), fig. 4.1.40) 2*. Plusieurs illustrations de la légende de Krṣna, aspect le plus souvent représenté de Viṣnu, ont également fourni loceasion de figurer le dieu armé d'une épée. Ainsi en est-il de la scène du meurtre de Kamsa, représentée pour la première fois sur lun des frontons du temple de: Banteay Srei (bibliothèque nord, fronton ouest : voir R(OVI:DA 2005, fig. 4.2.96) ${ }^{29}$. lin outre, un épisode, tiré cette lois-ci du Mahābhärata, ke Kirātārjunīya, a été illus-

27. Nous devons surseogir ici a une rnguete sur less divers fermes saluskrits ou khmers désignant des armes blanches.

28. Ine rée pourrat igalement ître identifice parmi ses attribuls sur dautres images on pierere de la diviniti. même s’il est souvent possible de la confondre aver une massue levéo. Kamaleswar Bhatlacharya 11961, p. 104, 107) pense. en revanche. pouvoir la reconnaître sur plusieurs images en brenze de Visnu - malheureuscment fragmentaires - publienes dans candis 1923. p. 22-24, pl. 3-2, $4-2$ at 5-1.

29. Pour dautres representations angkoriennes

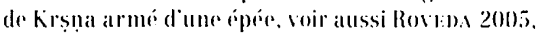
lig. 4.2 .99 lingkor lat : lour centrale. faree est. fronton inférieur) ot +.2 .107 (Angkor lat : pavillon diangle nord-ouest, mur sud). tré sur un des petits panneaux sculptés en bas-relief du Baphuon (gopura II ouest, ailo sud, face ouest) et montre Arjuna recevant l’arme páśupala - ici une épée - de Śiva. debout sur un piédestal (I.y Boreth 2003. p. 137, fig. 8 ; voir aussi Rovidd 2005. p. 168). Il s'agit là toutefois d'un cas à part où l'épée ne constitue pas à proprement parler un attribut du dieu. Inn gardant à l'esprit l'hypothèse de J. Boisselier, une autre divinité à retenir serait, en revanche, Durgā Mahișāsuramardinī (la « Destructrice du démon buffle », toujours figuréc à plusieurs bras avec souvent une épée dans l'une de ses mains. Si la plupart des images en rondebosse de la déesse sont préangkoriennes. cette divinité continue d'être représentée. mais essentiellement sur des bas-reliefs. à l'époque angkoriemne (BHatTacharya 1961, p. 91-92). Il convient de citer, une. nouvelle fois, le temple de Banteay Srei et un de ses célebbres frontons, où est sculptéo une Durgā Mahișāsuramardinī à huit bras. qui porte, entre autres armes, une épée dans sa main supérieure droite gopura I ouest, fronton ouest ; voir Rovi:dA 2005. fig. 4.5.94) $)^{30}$.

30. Il ne faut pas non plus oublier liconographio bouddhique khmore et, en particulier. les pre-

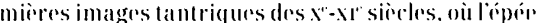
compte parfois parmi les attributs de lajrapani. ou peut-ître de Vajrin, une figuration préeoce de Ilevajra. De telles représentations dépées - ou, lout du moins de ceqüil semble raisommable dodentifier comme (les éperes - apparaissent, notamment, sur deux stôles bouddhiques (« raitya »), où Vajrapāṇi/ Vajrin est seulptí sur lune des lates à coté dautres divinites : la premiore stipe est conservere au muscis

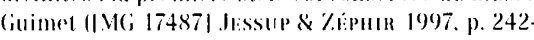

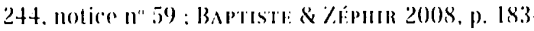
185, notice n" 521 la secende an musée national do Bangkok (Woomwala) 2007, p. 77-78, fig. 4-6). Par ailleurs. lépere est rgalement un attribut du bodhisattea lokeśrara. matis seulement dans sa forme "irradiante" "qui ne se developpe quatu rours du rigne de Jayavarman VIl (r. ens. 1181-1218: vraisemblablement dans les années 1190 . Il portio notamment col altribut dans sa main inférieure gatuche sur deux des fameux bas-relief's le representant à Banteay Chmar (Borssitols 1965, p. 77-78, lig. 2-3). Il ro est de même pour une série d'images en bronze. dont les mains en les attributs ont été próserves : une est conservere au Palais Royal de Phnom Penh l(c(t)i:s 1923, p. $46-47$, pl. 32) : une autre all musér (iuimet (V) 5940) : BAPTISTl \& KÉPIIIR 2008. p. $286-28 \pi$. notice n" 841 : ( enfin, trois autres statues.

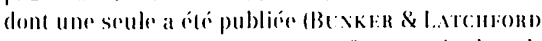
20(04. p. 278-281, notice n" 96). font partie de colIretions privines. 
Outre celle dattribut mobile, une autre fonction est également envisageable pour l'épée de Boston. Il n'est pas évident, en effet, que celle-ci ait été nécessairement portée par une statue, qu'elle ait été en pierre ou en bronze. Peut-être a-t-elle simplement été déposée à proximité de cette image de culte comme objet votif, ou encore offerte au Kamrateń Jagat mentionné dans l'inscription qu'elle porte et conservée parmi le mobilier de son sanctuaire. De telles offrandes d'armes faites à des divinités sont bien attestóes dans l'épigraphie khmère. Un exemple parmi d'autres est donné par linscription K. 1198, rédigée en sanskrit et en vieux khmer et datée du règne de Sūryavarman $\mathrm{I}^{\mathrm{N}}$, soit contemporaine de l'épée de Boston (voir supra fig. 4). Entre autres donations, elle mentionne notamment l'offrande par le Vrah Kạ̣sten 'Añ Śrì lakșmipativarma de deux épées (vieux khmer khān) au V. K. 'A. Śivalinga |Śival et de deux autres encore au dieu Nārāyaṇa IViṣnul (B, I. 34, 35 ; texte et trad. dans Pou 2001, p. 246, 251). Plusieurs exemples d'armes clairement votives sont, en outre, connus pour l'époque angkorienne, tel le fameux arc accompagné de sa flèche du musée Guimet (M 5990-5991) : à la fois en bronze et en fer, il est, lui aussi, de taille réduite - par rapport aux ares de combat des bas-reliefs - et daterait de la première moitié du xil" siècle (L.s BonmuUn 1994, p. 93-94 ; BAPTISTI: \& ZÉPHIR 2008, p. 368 , notice n" 114).

\section{Un bronze doré au mercure : héritages et pratiques ( $\mathrm{vI}^{\mathrm{C}} / \mathrm{VIII} \mathrm{I}^{\mathrm{e}}-\mathrm{XI}^{\mathrm{r}}$ siècles)}

lépée de Boston a été examinée au Scientific Research Laboratory du MFA en mars-avril 1969 et l'analyse de sa surface par spectrométrie d'émission atomique (NES) a permis didentifier des faibles traces d'or et surtout de mercure ${ }^{31}$. Celles-ci indi-

31. Iucune valeur quantitative nost toutefois donnée dans le rapport d'analyse. qui nöndique pas non plus la localisation procise des mesures larchives du Scientifie Research laboratory du Mlill. Par ailleurs, contrairement a ce qui est affirmé dans quent qu'une dorure au mercure, aussi dite à l'amalgame de mercure, a étć appliquée. sur le bronze lors du travail de finition.

E. Bunker \& I). latchford ont mis en parallèle ce résultat avec la date proposée par Long Seam (862 śaka, soit 940-41 de n. e..) pour faire de l'épée de Boston le premier bronze khmer doré au mercure clairement daté (voir supra p. 109). Selon ces mêmes auteurs, elle serait, en outre, à considérer comme l'une des preuves de la «popularité » quaurait connue cette technique de dorure sous le règne de Jayavarman IV (r. 921-41 de n. è. $)^{32}$. Seuls doux autres bronzes dorés au mercure et datés sur critères stylistiques de la même période, soit du style dit de Koh Ker (env. 921-45 de n. è.), confirmeraient toutefois cette hypothèse : une divinité fóminine sans provenance connue conservée au musée Guimet $(\mathrm{M} \Lambda 2239)^{33}$ et une tête fragmentaire de Garuda, elle aussi sans contexte archéologique, issue d'une collection privée ${ }^{34}$. Enfin, toujours à partir des trois mêmes exemples, l:. Bunker \& I). Latchford ont proposé de voir dans cette pratique de la dorure au mercure sous le règne de Jayavarman IV une pos-

Bowken \& 1.ATchlond 2008 (p. 45. n. 211, lobjet na jamais cele analyse par lluoreserence $X(X R)$.

32. "linhancing bronze objects by amalgam gilding appears to have beren quite popular during the

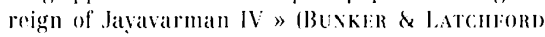
20(08. p. 45).

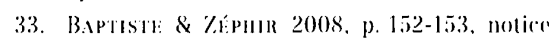
n" 44. Ia restauration de cette statuette en 1999 a refe loorasion de mettere en evidenere atu niveau de sa surface de tries importants vestiges de dorure. qui temoigneraient en laveur d'une dorure au mercure. In revanche, olle na jamais fait lobjet d'analyse par XRli, re quiont pourtant suggéré l:. Bunker $\&$ D. Latchlord Bunkik 2008 , p. 298 : Btwkt:k \& Latchlorle 2008, p. 45, n. 22). II est, en outre possible de se demander à quelle date a rete réalisese rette dorure. Ia statuette présente. en effet. la particularito d'être constiture d'un alliage a base de cuivere à tròs lorte teneur en argent $119 \%$ \% sans doute obtenu pour donmer une coloration brillante à sal surface (Bourciarte et al. 200)3. p. 111, 118 122. Appendix 21. Il serait ainsi convisageatble que la dorure alu mererure nait éte applicuereque dans un serond temps. à une date postrérieure.

34. BaxkR \& I.ATCHFORI) 2004, p. 435, n. 29.

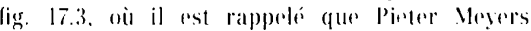
conservation scientist atl los Angeles county Yuseum of Art, a ramine la piece ct reconnu en surface une dorure au mercure : voir allussi Bexkle 2008, p. 298. sible influence d'artisans khmers provinciaux ou même d'artisans chams, tous venus travailler dans la nouvelle capitale du souverain, Chok Gargyar / I.ingapura (moderne Koh Ker) ${ }^{35}$. Cependant, outre les deux derniers bronzes mentionnés - l'épée de Boston, plus tardive, est évidemment à exclure de ce « groupe »-, aucune preuve historique ni témoignage archéologique no viennent à l'appui de cette nouvelle hypothèse.

Il convient néanmoins de s'aceorder avec ces auteurs pour reconnaître le hiatus existant - tout du moins en l'état actuel des recherches - entre l'apparition des premiers bronzes khmers dorés au mercure, sans doute entre le $\mathrm{vI}^{\prime \prime}$ et lo virr" siècle, et l'usage renouveló dont cette technique de dorure commencerait à faire l'objet à partir de la première moitié du x', ce qu'attestent seulement deux pièces datées du style de Koh Ker, comme nous venons de lo voir. Rappelons également que les premiers bronzes khmers dorés au mercure ne sont guère plus nombreux. Parmi eux se trouve un about de. hampe en forme do yakşa (?), peut-être du vi" siecle, qui provient des environs de Trà Vinh, dans le sud du Vietnam, et se trouve aujourd'hui au musée Guimet (MG 18318 ; BAPTISTIE \& ZÉPHIR 2008, p. $85-87$, notice n" 24 ; voir aussi BUNKFrR \& IATCHFORD 2004, p. 435 ; BUNKFR 2008, p. 297-298; BUNKER \& LATCHFORD 2008 , p. 27). Un autre exemple est fourni par une statue de Skanda, sans doute légèrement plus tardive (VII"-virI" siècle). Issue d'une collection privée, elle serait, elle aussi, originaire du Vietnam (BUNKreR \& I.ATCHFord 2004, p. 65-67 Inotice I" 13|, 435; BUNKER 2008, p. 297-298;

35. "Jayavarman IV apparently imported craftsmen from all over his kingdom and perhaps eren from champa to work at his capital. It is tempting to suggest that some of these imported craftsmen mat have brought knowledge of amalgam gilding with them, as there is little evidence of its use in khmer art during the ninth and tenth centuries until the Koh kor period " (BUNker 2008. p. 298 (nos italiques|) : voir aussi Bunker \& LATCHFolu) 20(04. p. 43.5 


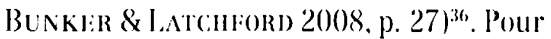
etre complets, il faut encore mentionner une statue fragmentaire de Buddha debout, récemment redatée du val" siècele (Woonward 20)10, p. 36, fig. 8) et conservée au musée national du cambodge à Phnom Penh (ga 5412) $)^{37}$. Provenant du Vat Banon, dans louest du Cambodge, elle relive sans doute d'une autre tradition technologique, qui aurait été toutefois contemporaine de celle plus méridionale représentée par les deux premieres pieces.

Toujours à propos des premiers bronzes khmers dorés au mereure, il est interessant de rappeler que plusieurs raisons laissent penser que le transfert de cette technique de dorure vers le Cambodge ancien aurait résultó dune influence du monde technologique chinois. Comme la récemment suggéré Paul Jott (2010)a, p. 86 : 2010b, p. 51), il se pourrait, en effet, que les premiers bronzes chinois dores au mercure "importés» en pays khmer, à dater des IV"-VII" siècles, aient joué un rôle dans ce transfert technologicue. Parmi les quelques exemples commus, tous sont bouddhiques. Iouis Malleret signale, le premier, une statuette de Buddha adossé dorée au mercure, où " il subsiste des traces cetendues de métal précieux" (1960, p. 201 notice n" 431, pl. 84-1 : voir aussi BLNKI:R 20(08, p. 301). Découverte dans la régrion dooc loo, dans le sud du Vietnam, celle-cise trouve aujourd'hui au muscé d'llistoire du Vietnam de Hô (hi Vinh-Ville. Vraisemblablement de même origine, une autrestaturterenservere au

36. I. premier bromer a diti analsse par PINi:

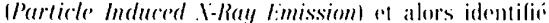

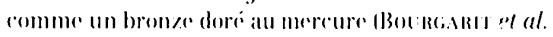

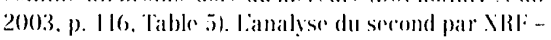

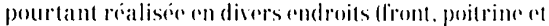
ventres - na pas permis, on revaluche. dobserver des traces de mereure au niseatu de sal surlatere liexaimen de celle-ci a nómonoms laisse penser à une dorure a lamalgame de mereure (rapport damalsise du lo juin 2003. arehives du Department of (conservation and

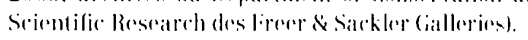

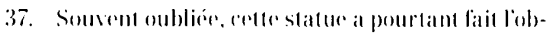

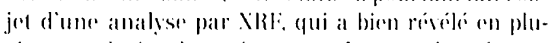

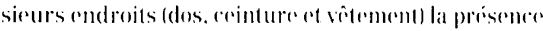

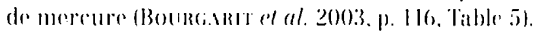

sein de ce musée et figurant cotte lois-ci un bodhisallea présente, quant à elle, de simples "traces de dorure » (MALILizi:T 1960, p. 208 notice n" 434|, pl. 4-3 ; voir aussi JI:TT 2010b, p. 51). Elle aussi aurait été toutefois dorée au mercure. Il est, en effet, possible de la rapprocher d'une statuette de bodhisattea tres similaire, qui a été récemment mise au jour avec un autre bronze chinois dans la région de Kompong (ham, dans l'est du Cambodge. ()r, selon P. Jetl (2010a, p. 8:3, 86, fig. 38. 38 a, 38b, $39 ; 2010 b$, p. 51, fig. 6-7 ; voir aussi BUNK 2008 , p. 301), ces deux pieces, entrées depuis dans les collections du musée de Phnom Penh (ga 6942 |bodhisalleal et 6943 | gardien|), ne pourraient etre autre chose que des bronzes dorés au mercure. Comme le souligne cet auteur, la dorure au mercure constitue en effet, à partir du II" siècle, un des traits caractéristiques des premiers bronzes bouddhiques chinois.

Par ailleurs, si seuls deux exemples de bronzes khmers dorés alu mercure sont attestés dans la premiere moitić du $x^{\prime \prime}$ siècle, cette technique de dorure serait. en revanche, mieux documentée à partir du siècele suivant. la datation ici proposéc pour l'épée de Boston (1040-41 de n. i.) semble donc davantage en aceord avec l'histoire des techniques telle qu'elle nous est connue pour le XI" siècle. De cette époque date, notamment, une série de statues en bronze-dont certaines monumentales -, toutes attribuables au style du Baphuon (env. 1010-80 de n. e.). qui se caractérisent par d’importants vestiges de dorure visibles au niveau de leur surface et témoignant, là encore, en faveur d'une dorure au mercure. Plusieurs de ces pièces sont déjà citées dans Bunki:R \& LaTCHroRD 2008 (p. 45, 56, fig. 4.17, 4.19, 4.21a-b) $)^{3 \times}$. la

38. Il sagit du Herajra de Banteay Kderi. conservé au

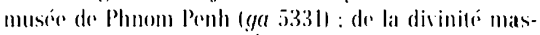
ruline (Xandikeśsara ou Śiva ?) du P'rasal kamphaneng Yai. conservere alu musce national de Plimai : enfin. de doux autres divinités masculines (Śiva ?. respertive-

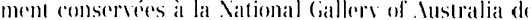

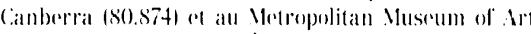

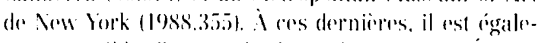
ment pessible dajouter la divinite masculine (Siva ?

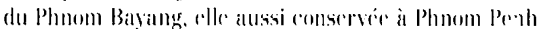

question d'une dorure au mercure pourrait, en outre, se poser pour dautres statues de même tradition stylistique dont la surface présente ou a présenté des traces de dorure, certes plus réduites ${ }^{39}$. Infin, il n'est pas inutile de rappeler que, parmi toutes les pieces de cette série, une seule a fait l'objet d'analyses - très certainement par XRL1:-dont les résultats ont montré des traces de mercure en divers endroits : il sagit d'une divinité féminine conservée au (Geveland Museum of Art (1982.51), dont le diadème-couronne, le sampot et tous les éléments de parure ont été dorés au mercure (STRAHAN \& MaINl:s 2000), p. 199. n. 2. citó dans Bunki:R \& I.ATCHFORD 2004. p. 241-243, notice n" 8.3$)^{\text {t+1" }}$.

En se fondant essentiellement sur des criteres stylistiques, plusicurs historiens de lart ont avancé l'hypothèse d'une même école de bronziers, qui aurait produit lensemble de cette statuairet1. Attestée dos les

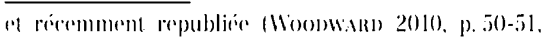
fig. 20. 20)a ; (juy 2010, p. 103. 106, fig. 31 ; voir alussi

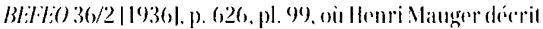

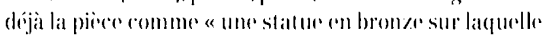

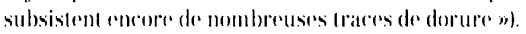

39. II en est ainsi pour lo fiamerux viṣ!nu Anantaśăịin du Mebon occidemtal, dont le buste re plusieurs autres fragments sont conserves all musce do Phomom Pronh lea 2084, 2988.1. 2988.3.

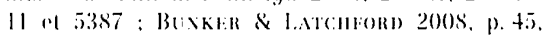
fig. t.ls. 4.20). Au moins deux atutres statues seraient regalement a remenir : lo Sisal du Vat Por laboeruk. Iui aussi conserve a Phomom Penh en plusieurs fragments lya 2726-2731 : woir fiches 1$)($ )

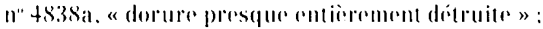

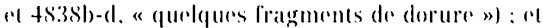
une divinite matsculine (Viṣnu ?) du musce ciuimet

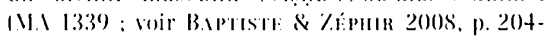
205. notice n" 39 , "Bromze, draces de dorure"

40. lin revanche, si la divinite massulime du

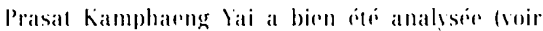
Warângkhana ÎAvkso 1992. p. 37-3si. attcune

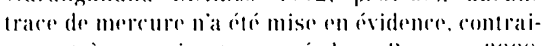
rement à re qui est alvamer dans Buthl:k 2008. p. 299. II ne laut pas non plus oublior la divinite masculine du muscé (iuimel (N1:1339). dont la surfare a cle. clle anssi. analysce - par PIXl: - sans gur des traces sulfisantes de mereure aiemt dé toutefois identilieres. ere qui ne weut pas dire pour autant que la statue nétail pas dorre alu mereure

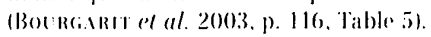

41. Ine liste plus ou moins exhaustive de cess sta-

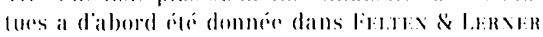
1989, p. 224-227 : voir aussi Woomward 20(0)3.

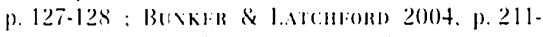

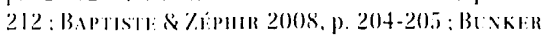

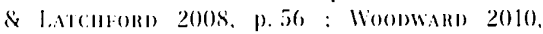
p. $50-51:(3012010)$, p. 106. 
années 1040, celle-ci aurait participé à la politique édilitaire du souverain Udayādityavarman II (r. 1050-1066 de n. e.), son activité se prolongeant encore jusquaux années 1180 et au-delà. Au vu du caractère mobile de ces images - à l'exception notable du Viṣnu du Mebon occidental -, aucun consensus n'a toutefois été trouvé quant à la localisation du ou des ateliers de bronziers correspondant à cette production. D'autres

\section{Bibliographie}

Irchiress

DCA bépôt de la Conservation dingkor. Fichier dinventaire. Paris : Archives de libole francaise d'lixtreme-()rient.

Jl: 1909-1955: Journaux de fouilles. Paris, Archives de l'École française d'lixtreme-()rient.

RCA. I908-1973: Rapports de la Conserenation dingker, Paris, Arehieses de lificole francaise d'lixtrême-()rient.

Lillerature secondaire

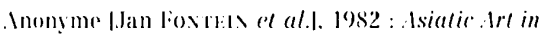
the Vuserum of line. Irts, Bostom. Boston (.Matss.l. Yuseum of line Irts.

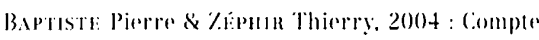
rendu de Buxkl: \& I.ATChFord, Arts asialiques 59, p. 182-184.

- 2008: liart khmer dums less collections du musée cinimet. Paris. Réunion des musie's nationaux.

Biry Pierre. 2007 : "L.es couteaux angkoriens do Trapeang Thlok el de Prasal Trapeang Ropou ". BIIT:(0)9. p. 95-110.

Buntacmarsa Kamaleswar. 1961 : le's religions brahmaniques dans l'ancien Cimbedge d'apro's

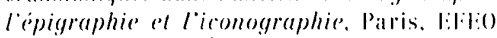
(l'ublications de l'licole françatise dilextrome(Orient +9)

Bolssi:11:R Jean, 196.5: "Precisions sur quelques images khmeres d Walokitessara. les batsreliefs de Bantas Chmalr". Irls asialiques 11/1. p. 73.59

- 1960 : 1sie de Suel-1st I. Ie Combodge. Paris 1. al I. Picard et C." Mamuel d'archeologie d'ixtrimo-()rients. criteres, notamment techniques. seraient certainement à prendre en compte pour identifier uno telle école ou de tels ateliers et la technique de la dorure au mercure, au même titre que celle-souvent citée-des incrustations. pourrait constituer l'un d'entre eux.

Avec: l'épée de Boston, un nouvel exemple de bronze khmer doré au mercure, analysé et clairement daté, peut désormais être ajouté à cette production du xI" siècle. qui a dû fournir également pour les divers sanctuaires du royaume angkorien tout un ensemble dobjets rituels en bromze, dont seule une infime partie nous est malheureusement connue.

Gerdi Gerschheimer \& Brice Vincent ${ }^{42}$

42. Cierdi ciersehheimer est directeur doutudes à lícole pratique des hatutes citudes : Brice Vincent est dectomant a liuniersile de Paris 3 -Sorbonne Vouvelle.
- 1972: "Travaux de la Mission archóolosique francaise en Thailande fuille-november 1966 ". Arts asiatique's 25, p. 27-9).

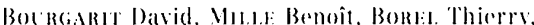

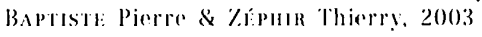
"A Millenium of Khmer Bronze Melallurgy: Analycical studies of Bronge Artilacts from the Musée Guimet and the Phnom Penh Vationa Museum ". in .J:TT Paul Red.). Scientifir Rese'arch in the Fïeld of Asian Art: Procesedings of the lïrst forbes Symposium at the freer ciallery of art. london, . Irehelype P'ublications, p. 103-126.

Bexken l:mma (... 2008 : "Amalgam (Gilding in Khmor Culturem. in Baces l:lisabeth. A. (itortek

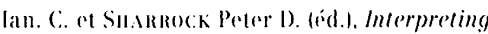
Soulheast Isia's Past. Vomument, Image and Text. Singapore. Nus press, p. 296-305.

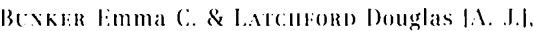
2o(1) : Idoration and filory: The Golden lge of Khmer Irt. Chicago. Art Media Resources.

- 2oos : Khmer cold: Gifts for the Gods. Chirago: Irt Vedia Resolures.

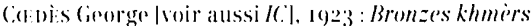
Eucle basee sur des documents recueillis par 11 P. I. ferere-Pontalis dans les collections publique's "l priceres de Bangkok el sur les piecess conser reses au P'alais Royal de Phom Penh, au Museo

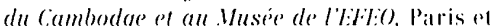
Bruxelles. G. Van Oest (Ars Asiatica

- 1061 : " les expressions erah kamrateri an ot kamraleni jagal all vieux-khmer" ". Adyar library Bulletin 25, p. $4+7 \cdot 460$

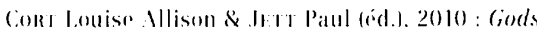
of Ingkor: Bromzes from the Vational luse'um of Cambodia. Mashington, I nisersits of Nashington P'ress.
Colr BIx Paul. 1988: "la louillo du Sras-Srang" in

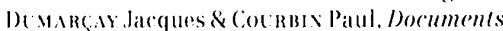
graphiques de la Conservation d:Ingkor 19631973. Paris lablio (Collection de textes en doenments sur l'Indochine 17). p. 21-44.

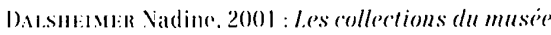
national de Phom Penh. L'art du Cambodge ancien taver une introd de Brumo Dacitsst.

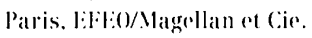

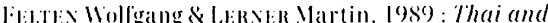
cambodian Sculpture from the oth to the 1tth Centurie's. Iondon, Philip Wilson Publishers.

FII.Io\% Jean. I980: " Sur Je civaïsme a le bouddhisme du Cambodge. a propos do deux livres

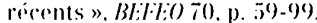

lixor l.ouis. 1928 : "Nouvolles inseriptions du Cambodge III. l,a stiple du Pràsist Tripãin Ruul ". BBLF:O() 28/1, p. $58-80$

Gostank Cirorge, 1921 : Recherches sur les cambodgiens, d'apress les lextes ef les monumonls depuis les premiers siede's de notre ine Paris. A. Challamel.

IC 1937-66: Cands Georoce Inscriptions du Cambodge. \& vol., Hanoi/Paris, l:Flo (Collertions de textes el doruments sur l'Indochine 3 ).

(ity John. 2010): Angkerian Vetalwork in the Tromple Selting: leons. Arehitectural Adornment. and Ritual l'araphernalia ", in (Core \& I:TT féd.) 2010. p. 8 s-1209.

Jace-H1:Bcosacin Vichel. 1979: larmement ol

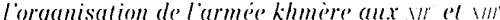
siirle's dapres le's bas-reliefs dingkor lat. du Baygon et de Bemeray C.hmar. Paris, Presse's unirersitaires de liance APublications du muse

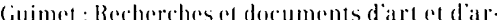
rhrologic 121. 
Jucouss claude, 1971 : "Supplement au lome: VIII des Inscriptions du Cambodge ". BlFFio ss. p. $177-195$.

- 1977-78: "LRapport sur les conlifrences de lan-

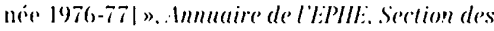
scionces historiques et philologiques, $1977-7 s$ p. $1067-1080$.

- 1994: " les kamralen jagal dans laneien Cambodge m, dans BazoT lirancois delir.l. Recherches nourelless sur le Cambodge (Paris: 1:1:1:(0). p. 213-22:5.

J1:Nen Philip N., 2009 : A Dictionary of Angkorian khmer. Cauberra, l'acific linguistics. Rese arel sehool of Pacific and Asian studies, The Australian Vational Iniversily.

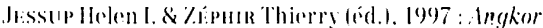
e' dix siecles d'art khmer. Paris, Réunion des Muscés Nationaux.

J1:11 P'aul, 2010ai: "A Trehnical Study of the Kampong (ham figure Group m, dans conT \& JETT (rid.) 2010, p. $78-8 \bar{i}$.

- 2010b) : Buddhist Bronzes in Cambodia: Newly Discovered Cache ". Orientalions $+1 / 5$. p. $48-52$.

KI.riblin cieorgess 1981 : Problemes de reference descriptions definies et noms propres. [Metel. Centre d'analyse sontaxicue de l'universiti de Netz IRecherehes linguistiques 61.

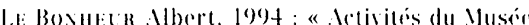
national des lrts asiatiques - Guimet Irquisitions. Isie du Sud-Iist ". Irts asiatiques 49. p. $93-96$.

I.r Boreth, 2003 : "Nalrating the Deaths of Dronil and Bhurisravats al the Baphuon ". Ir/s asia tiques 58, p. 134-137.

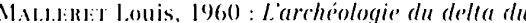

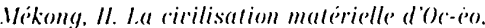

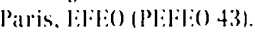

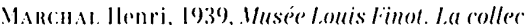
lion khmere. Ilanoi. H:Fl:

Portat:n (hristophe (dir.), 2007 : Vission archeoso gigue franco-k-kmere' sur laménageme'nt duterritoire angkorien (11:1/\%:1T:1). Campayne 2000 Rapport. Sirm Roap, flili:()

Pot Saveros, 2001 : Aourelles inscriplions de Cambodge // el III. traduites a ciditóes. Paris.

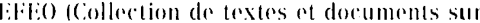
Jndochine 22 et $2: 31$

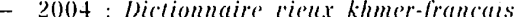
anglais / in old khmer-lirench-linglish dictonary. Paris/Budapest/Torino. LHarmattan.

Rovina Vittorio, 2005: Images of the Gods. Khmer bytholog!y in Cambodia. Laos \& Thailand. Bangkok. River Books.
Sortur pominicue 2008 : "Denomber les biens de dieu : clude de la numiration du vieux khmer (va"-xu" siocles saka)". Siksacake 10, p. jl-so |version francaisel. p. 172-206 |rersion khmere. trad. par Vichel Antelmel.

- 2009 : "Organisation religieuse et profano du temple khmer du vel" au XII" sierele ". 3 vol.

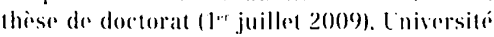
Sorbenne mouverlle Paris 3.

Stranax Domna K. \& Maves Christopher A. 2000 : "Lacequer as an Adhesive For (iilding on Copper Alloy Sculpture in Southeast Asia ", dans Draymax-Wiassi:n Terry féd.), Gilded Metals. Iistory. Terchnology and Conserratiom. I.ondon. Arehetype publications, p. 185-201.

Warângkhana ÎAwkxo. 1992 : "Kân 'anurak pratimâkam samrit phop na Prâsâl Sa Kampharngr Yai Conservalion of Bronze Seulpture from Prasal Kamphaeng Yai, Amphoe l thumphon Phisai. (hangwat Si sa koll ". Silpakorn Journal $35 / 4$ p. 16.-3s.

Noomwas) Hiram W. J., 2003: The Irl and Archilecture of Thailand, I, eiden \& Boston. 1:. I. Brill.

- 2007: "The Karandarsuha Sulra and Buddhis Arl in loth-century Cambodia ", dans Pai. Pratalpadilya tod.). Buddhist $1 \mathrm{rt}$. Form \& Meaning, Mumbai, Marg, p. 70-83.

- 2010 : "Bronze Seulptures of Ancient Cambodia ", dans cont \& JatT téd.) 2010. p. 3() .77 